\title{
Patrones de uso de Internet en población universitaria española
}

\author{
Marina Julia Muñoz-Rivas*, Ma Eugenia Navarro Perales**, Natalia Ortega de Pablo*** \\ * Universidad Autónoma de Madrid \\ * Universidad Complutense de Madrid \\ *** Centro de Enseñanza Superior San Pablo-CEU \\ Enviar correspondencia: \\ Departamento de Psicología Biológica y de la Salud. Facultad de Psicología. Ciudad Universitaria de Cantoblanco. 28049 Madrid. \\ Universidad Autónoma de Madrid. Telf.: 91.397-87-51. E-mail: marina.munoz@uam.es
}

\section{RESUMEN}

En el presente estudio, se analizan los patrones de uso de Internet y de sus recursos específicos como páginas web, correo electrónico y chats específicos. Para ello se ha contado con una muestra de 1301 estudiantes universitarios de ambos sexos y se analizaron las características sociodemográficas, los parámetros de uso de Internet de forma global y específica, los motivos y la interferencia del uso en la vida cotidiana de los sujetos.

Los resultados muestran que el 33,9\% de la muestra lleva conectándose a la red entre 1 y 2 años y el 23,1\% afirma hacerlo en un intervalo de entre 2 y 5 años; el 43,3\% de los universitarios del estudio prefiere conectarse por las tardes, el 39\% por las noches y el $17,7 \%$ por las mañanas haciéndolo generalmente desde su domicilio. Un alto porcentaje de la muestra realiza un uso moderado de Internet al analizar el tiempo de conexión (invierten menos de 4 horas semanales) y tan sólo un 1\% afirma invertir un mínimo de 5 horas diarias durante los siete días de la semana y sólo el 17\% afirma percibir algún tipo de interferencia utilizando los recursos de Internet en su vida cotidiana. En cuanto a la variable sexo son los hombres los que dedican más tiempo a la semana en la utilización de cualquiera de los recursos de Internet.

En general, los estudiantes encuestados realizan un uso muy moderado de Internet dedicando un promedio semanal que va de 11 a 20 horas lo que nos indicaría que no hay un uso abusivo o patológico cercano a la adicción, siendo un grupo reducido de jóvenes los que sí estarían próximos a este tipo de psicopatología.

Palabras clave: Internet, estudiantes universitarios, uso, abuso

\section{ABSTRACT}

In this study, the Internet usage patterns and resources, such as websites, e-mail, and specific chats, were analysed in a sample of 1301 university students of both sexes. Their sociodemographic characteristics, global and specific Internet usage parameters, motives and the interference of such use in their daily lives were studied.

The results show that $33.9 \%$ of the sample had been using the Internet between 1 and 3 years, and $23.1 \%$ reported using Internet for a period ranging between 2 and 5 years; $43.3 \%$ of the university students preferred connecting to Internet in the afternoon, 39\% at night, and $17.7 \%$ in the morning, generally from home. On analyzing connection duration, a high percentage of the sample made moderate use of Internet (with less than 4 hours per week), and only $1 \%$ reported spending a minimum of 5 hours per day, seven days a week, and only $17 \%$ admitted perceiving some kind of interference in their daily lives from the use of Internet resources. By gender, men spend more time per week than women using any of the Internet resources.

In general, the students surveyed use the Internet very moderately, devoting a average weekly period ranging from 11 to 20 hours, which would indicate that there is no abusive or pathological use, approaching addiction, of the Internet. Only a reduced group of young people came near to this kind of psychopathology.

Key words: Internet, use, students, university, abuse.

\section{INTRODUCCIÓN}

A ctualmente, vivimos en una época de cambios tecnológicos rápidos. Concretamente, desde la década de los 80 , ha habido una importante revolución tecnológica con la aparición de sofisticados ordenadores y de Internet. En este sentido, en países como Estados Unidos, ha crecido notablemente el número de familias que poseen ordenadores en casa pasando de un 7\% en 1983 a un 44\% en 1997 (Tapscott, 1998). En lo que respecta a Internet, en 1980, el acceso a Internet estaba limitado a un selecto grupo de científicos y académicos pero, en menos de dos 
décadas, ha sufrido un crecimiento exponencial llegando a acceder regularmente a este recurso en el año 1999, el 37,4\% de la población general de este país (101 millones de personas) (NielsenNetRating, 1999). Este importante incremento se ha evidenciado así mismo en la población universitaria en donde el acceso a Internet por parte de los alumno/as, se ha incrementado notablemente en la última década (NCES, 1999; Schumacher y Morahan-Martin, 2001).

En nuestro país, según los datos de la Encuesta General de Medios, el uso de Internet se ha incrementado de un 0,7\% en 1996 a un 10,5\% en el año 1999 (Estudio General de Medios, 1999). Estos resultados muestran la paulatina evolución del uso de Internet en España y señalan un aumento progresivo en los próximos años. Los datos obtenidos a través de la mencionada encuesta sobre el uso de Internet nos muestran, además, que el perfil del "navegante" español es un varón cuya edad oscila entre los 20 y los 35 años, con un nivel académico secundario y/o superior, que habitualmente accede a la red desde su domicilio con una frecuencia semanal, llegando a estar conectado a la red una media de 10 horas. Además, entre 1996 y 2000, se ha evidenciado la incorporación masiva de usuarios menores de 20 años que pasan, de una participación casi simbólica en 1996, a constituir una quinta parte del conjunto de navegantes en el año 1999 (Estudio General de Medios, 1999).

Es indudable que Internet, como medio de comunicación, de ocio y de obtención de información se está convirtiendo en una herramienta necesaria en la sociedad occidental. El acceso a Internet provee información sobre multitud de áreas. En 1997, la American Internet User Survey reflejó que el $87 \%$ de los usuarios de Internet consideraban que la red les proporcionaba un acceso eficaz a la información que necesitaban diariamente $y$, el 77\% de los encuestados, afirmó que estar en la red les había ayudado considerablemente a ser más eficaces en su trabajo (FIND/SVP, 1997).

Teniendo presentes los aspectos señalados sobre su clara utilidad, resulta obvio que la irrupción de estas nuevas tecnologías ha de tener algún efecto sobre la sociedad en general y sobre la conducta de los individuos en particular, en función del uso que se haga de estos servicios (Anderson, Law y Mitchel, 1995; King y Kraemer, 1995).

Muchos son los profesionales dedicados a estudiar este fenómeno que coinciden en afirmar que, sin lugar a dudas, Internet está transformando la vida económica y social, aunque se muestran en desacuerdo acerca de la naturaleza de los cambios que produce y de su dirección beneficiosa o perjudicial. En este sentido, la investigación científica ha mostrado, por un lado, el efecto positivo de Internet sobre la autoestima en grupos que poseen características marginadas por la sociedad (McKenna y Bargh, 1998) y sobre el positi- vo desarrollo de relaciones interpersonales a través de la red (Parks y Floyd, 1996). Por otro, diversos estudios señalan los efectos adversos de su uso sobre el bienestar psicológico y la implicación social de los que la utilizan (Kraut et al., 1998), señalando la existencia de relevantes signos de interferencia derivados del mismo (Griffits, 1997).

En el momento actual, son muchas las especulaciones teóricas y estudios científicos que se centran en el estudio del uso patológico de Internet, planteando la posibilidad de que su uso y/o abuso pueda llegar a generar adicción (Brenner, 1997; Echeburúa, Amor y Cenea, 1998; Young, 1998), si se consideran determinados componentes conductuales similares al de otro tipo de adicciones (Young, 1998) y las consecuencias negativas para la vida cotidiana (Davis, Smith, Rodrigue y Pulvers, 1999).

En cualquier caso, consideramos que el paso previo a realizar este tipo de afirmaciones y los modelos teóricos que se derivan de ellas, es delimitar el uso que se realiza de este recurso en un amplio espectro de poblaciones. Por este motivo, parece necesario desarrollar estudios que exploren la realidad del fenómeno para así, desde una posición real y objetiva, desarrollar modelos teóricos sobre el diagnóstico y la posible adicción patológica a este tipo de recursos.

Así pues, y atendiendo a la escasez señalada anteriormente de análisis preliminares sobre la forma en la que es utilizado este recurso en nuestro país, el objetivo del presente estudio fue explorar detalladamente los patrones de uso y/o abuso de Internet en una amplia muestra de estudiantes universitarios, examinando la existencia de diferencias en función del sexo y la edad en cuanto al uso de los diferentes recursos de la red, con el fin de ofrecer resultados empíricos que sitúen a los profesionales interesados en este campo ante una aproximación realista de la situación actual en nuestros jóvenes.

\section{MATERIALY MÉTODO}

\section{Muestra}

Para el presente estudio, se contó con una muestra total de 1.346 estudiantes universitarios de diferentes licenciaturas provenientes del Centro de Enseñanza Superior San Pablo-CEU y de la Universidad Complutense de Madrid.

Dentro de cada uno de los centros y licenciaturas el muestreo se realizó por conglomerados tomando el aula como unidad muestral. Las aulas participantes fueron seleccionadas al azar tomando en consideración la disponibilidad de los profesores y de los alumnos dependiendo del calendario del programa docente. 
Todos los sujetos participaron de forma voluntaria en el desarrollo de la prueba y eran conocedores de que ésta formaba parte de una investigación sobre los patrones de uso de Internet realizada desde el Centro de Enseñanza Superior San Pablo-CEU.

\section{Instrumento de evaluación}

Considerando previamente otras encuestas sobre el uso de Internet existentes en la literatura científica sobre este área y ante la ausencia de protocolos validados para la exploración del patrón de uso/abuso de Internet, se desarrolló una encuesta compuesta por 19 ítems categóricos y 69 ítems dicotómicos (con opciones de respuesta verdadero/falso), que recoge información sobre diversos aspectos como datos demográficos, parámetros de uso de Internet tanto de forma global como de utilización específica de los diferentes recursos que ofrece, motivos para utilizarlos, relaciones sociales a través de la red e indicadores de uso patológico de Internet. El único criterio de inclusión de ítems y variables durante el desarrollo del instrumento fue el criterio de validez aparente.

Concretamente, las variables objeto de estudio del presente trabajo fueron:

a) Características demográficas. Se analizaron a través de 6 cuestiones que analizaban el género, la edad, el tipo de estudios universitarios que se realizan, el estado civil y la situación laboral de los encuestados.

b) Parámetros de uso de Internet de forma global. Fueron evaluados a través de 6 ítems categóricos que hacían referencia al tiempo que se lleva utilizando Internet, la frecuencia semanal con la que se accede a la red, el lugar y el momento desde el que se produce la conexión y la pertenencia a organizaciones dentro y fuera de Internet.

c) Parámetros de uso de recursos específicos en Internet. Se analizaron a través de 6 cuestiones referidas al uso de recursos específicos de la red: correo electrónico, acceso a páginas web, chats, foros de discusión, Protocolos para Transferencias de Ficheros (FTP) y juegos en red.

d) Motivos para el uso de Internet. Los encuestados debían señalar los motivos por los cuales utilizaban Internet escogiendo entre 10 razones específicas: realización de tareas académicas, crecimiento y desarrollo personal, descarga de programas, comunicación con amigos, contacto con gente nueva, comunicación con personas con las que se comparten intereses, acceso a información de áreas de interés personal, compras en red, acceso a cibersexo y otros motivos.

e) Interferencia del uso de Internet en la vida cotidiana. Se evaluó a través de 19 ítems dicotómicos que incluían los siguientes signos de interferencia: recibir facturas de al menos el doble de lo esperado, tener deudas derivadas de los honorarios de conexión, haber solicitado préstamos para cubrir los gastos de conexión, tener problemas familiares por el uso de Internet, llegar tarde a citas por estar conectado a la red, modificación de planes sociales para seguir conectado, preferir pasar una tarde en Internet a salir con un amigo, discusiones derivadas del uso abusivo de Internet, problemas con amigos por el uso de la red, pérdida de amistades por pasar mucho tiempo conectado, disminución de las calificaciones académicas, absentismo escolar, problemas con los superiores derivados de la implicación en la red, deterioro del trabajo y/o del rendimiento, problemas en el trabajo o los estudios por el uso de Internet, descuido de las obligaciones, renuncia o reducción de actividades importantes, abandono o reducción de actividades de ocio habituales y reiteración en el uso a pesar de los problemas experimentados.

\section{RESULTADOS}

\section{Características de la muestra}

De las 1.346 encuestas recogidas, antes de realizar los análisis de datos oportunos, se procedió a eliminar todas aquellas en las que quedaban incompletos los datos demográficos y aquellas en las que el examinado había dejado más del 10\% de los ítems sin responder. Así pues, para el presente estudio se contó con una muestra total de 1.301 sujetos de ambos sexos, siendo el $28,7 \%$ varones y $71,3 \%$ mujeres.

El rango de edad de los individuos de la muestra osciló entre los 18 y los 30 años, quedando situada la media de edad en los 20,4 años.

En cuanto al estado civil, el 99,3\% de los individuos afirmaron ser solteros, el 0,2\% estar casados y sólo el 0,5\% estar divorciados.

Al considerar su situación laboral, un alto porcentaje de los universitarios encuestados afirmaron dedicarse exclusivamente a completar sus estudios $(90,4 \%)$, mientras que el 9,6\% manifestaron compatibilizar sus estudios con un empleo.

Atendiendo al tipo de estudios realizados (véase, figura 1), la muestra estuvo compuesta mayoritariamente por alumnos de las licenciaturas de periodismo $(37,7 \%)$, psicología $(24,7 \%)$, biblioteconomía y documentación $(17,7 \%)$ y publicidad $(11,2 \%)$ y, en menor porcentaje, por estudiantes de medicina $(7,4 \%)$, derecho $(1,1 \%)$ y comunicación audiovisual $(0,2 \%)$.

\section{Parámetros de uso de Internet}

Del total de la muestra analizada, el 57\% de los estudiantes afirman ser usuarios de Internet desde hace varios años, concretamente, el 33,9\% lleva 
Figura 1. Distribución de la muestra en función del tipo de estudios

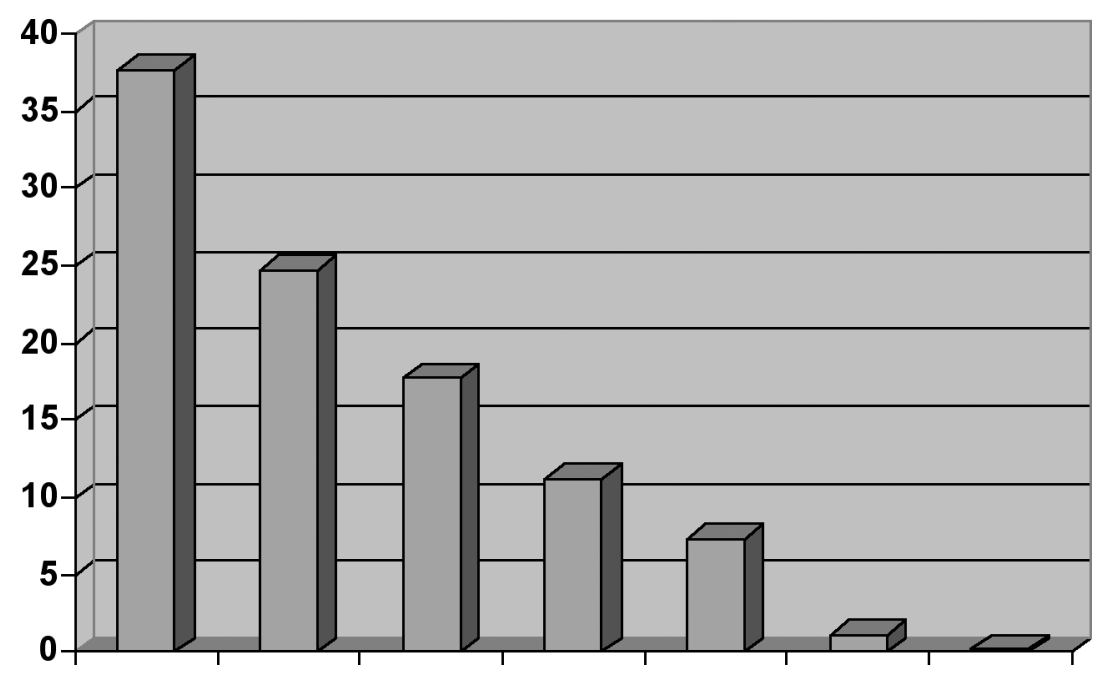

Figura 2. Distribución de la muestra en función del tiempo transcurrido desde la primera conexión a Internet

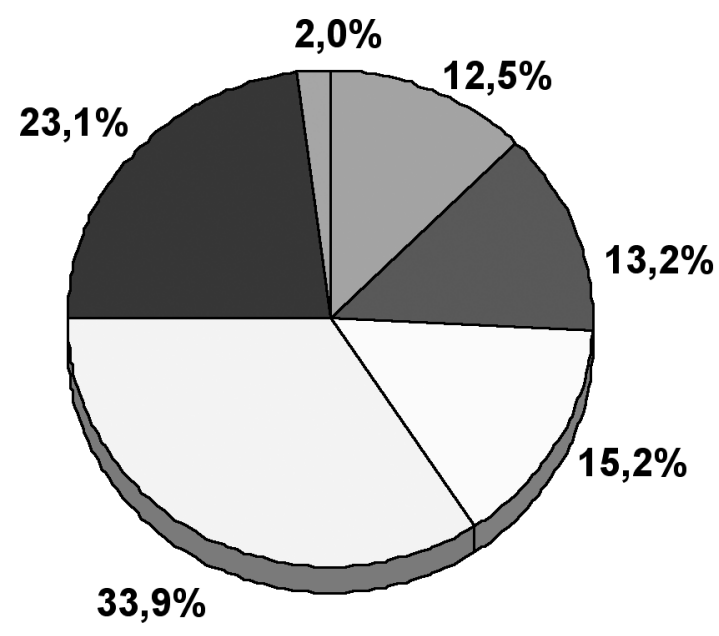

\begin{tabular}{|l|}
\hline$\square$ 0-3 Meses \\
$\square$ 4-6 Meses \\
$\square$ 7-12 Meses \\
$\square$ 1-2 Años \\
$\square$ 2-5 Años \\
$\square$ Más de 5 Años \\
\hline
\end{tabular}

conectándose a la red entre 1 o 2 años y el 23,1\% afirma venir haciéndolo en un intervalo temporal de entre 2 y 5 años (figura 2).

Mayoritariamente, la conexión se realiza desde el domicilio particular $(62,9 \%$ de la muestra), aunque prácticamente el $31 \%$ de los encuestados afirma hacerlo desde su centro universitario. En cuanto al momento del día en el que utilizan más frecuentemente Internet, el 43,3\% de los universitarios encuestados prefiere conectarse por las tardes, el 39\% por las noches y el $17,7 \%$, lo hace por las mañanas.
Las dos dimensiones que definen el patrón de utilización de un ordenador doméstico son la cantidad de tiempo dedicado y la diversidad de usos, siendo esperable una evolución tendente a la ampliación del número de utilizaciones diferentes, lo que supondrá un incremento en el tiempo total de utilización.

En cuanto a la primera de las dimensiones, tal y como se observa en la figura 3, en nuestra muestra, la cantidad de tiempo semanal que más frecuentemente se invierte en la utilización de Internet es de 0 a 2 horas (el $52,5 \%$ de los jóvenes) y de 3 a 6 horas (el $27,6 \%$ ), disminuyendo considerablemente el porcenta- 


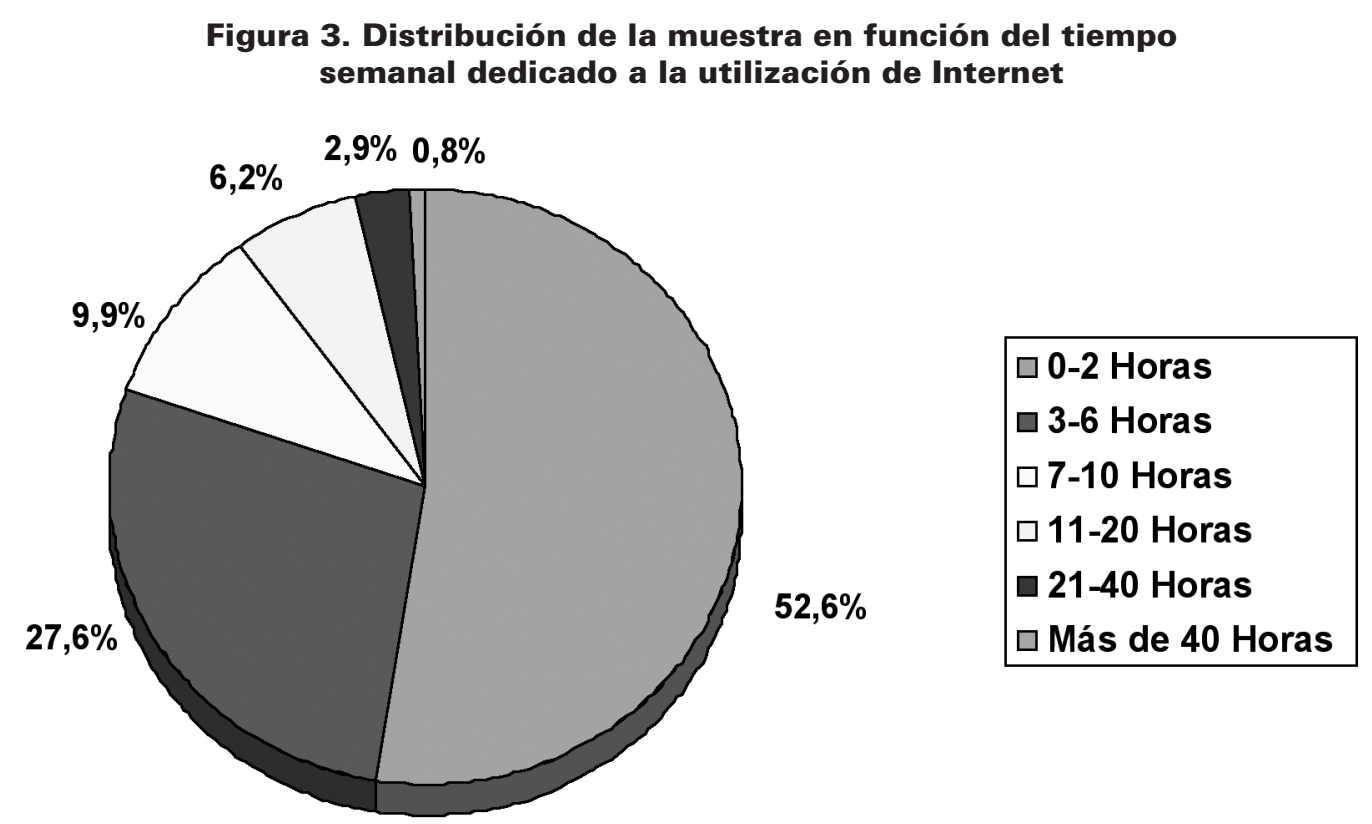

je de usuarios que hacen un uso de la red durante un tiempo más prolongado.

Al analizar la segunda de las dimensiones propuestas, cruzando el tiempo que los universitarios invierten en la red y el tipo de actividades que realizan cuando están conectados (tabla 1), se observa que el recurso más utilizado y en el que se invierten más horas a la semana es el acceso a páginas web (prácticamente, el $77 \%$ de la muestra), seguido del correo electrónico (el $75 \%$ de los encuestados) y de las líneas de chats $(37,4 \%)$. Los recursos menos utilizados son los Protocolos para Transferencia de Ficheros, los juegos en red y los foros de discusión, que cuentan con el 21,9\%, el $16,8 \%$ y el $15,2 \%$ de usuarios, respectivamente.

Con respecto a la participación en clubs u organizaciones tanto dentro como fuera de Internet (tabla 2), los datos muestran que la mayor parte de los estudiantes universitarios encuestados participan en uno ó dos clubs tanto fuera de Internet como dentro de Internet.
El tipo de clubs fuera de Internet a los que más frecuentemente se asocian son organizaciones deportivas (19,8\%); mientras que dentro de Internet son las organizaciones de tipo cultural, social y las de índole no política, deportiva ni solidaria las preferidas por los estudiantes (prácticamente el $41 \%$ de la muestra).

Finalmente, al estudiar los motivos que alegan los universitarios para el uso de Internet cabe señalar que las razones más frecuentemente argumentadas para su utilización fueron: la realización de tareas académicas (28\%), el mantenerse informados sobre nuevos desarrollos en áreas de interés personal (18\%), el poder hablar con amigos $(17,4 \%)$, el propio crecimiento y desarrollo personal $(11,5 \%)$ y, por último, el poder descargar programas informáticos (7,6\%). Por el contrario, los motivos menos frecuentes resultaron ser el conocer a gente nueva $(5,8 \%)$, hablar con otras personas con las que se comparten intereses $(3,4 \%)$, acceder al cibersexo $(3,1 \%)$ y hacer compras $(1,7 \%)$.

Tabla 1. Porcentajes de usuarios en función del tiempo semanal dedicado a la utilización de los diferentes recursos que ofrece Internet

\begin{tabular}{|lccccc|} 
RECURSOS & O Horas & $1-2$ Horas & 3-5 Horas & 6-15 Horas & Más de 15 Horas \\
\hline Correo Electrónico & 24,9 & 51,8 & 17,7 & 3,8 & 1,8 \\
\hline Páginas Web & 23,1 & 40,6 & 22,4 & 7,1 & 6,8 \\
\hline Chats & 62,6 & 26,9 & 6,9 & 2,8 & 0,8 \\
\hline Foros & 84,9 & 12,7 & 1,5 & 0,5 & 0,4 \\
\hline Protocolos para Transferencia de Ficheros (FTP) & 78,1 & 15,8 & 3,6 & 1,3 & 1,2 \\
\hline Juegos en red & 83,2 & 12,9 & 2,6 & 1,1 & 0,2 \\
\end{tabular}




\begin{tabular}{|lcc|}
\hline $\begin{array}{c}\text { Tabla 2. Distribución de la muestra en función de la participación } \\
\text { en clubs u organizaciones fuera y dentro de Internet }\end{array}$ \\
N ${ }^{\circ}$ DE CLUBS U ORGANIZACIONES & FUERA DE INTERNET & DENTRO DE INTERNET \\
\hline $1-2$ & 87,9 & 88,7 \\
\hline $3-5$ & 11,1 & 9,8 \\
\hline $6-9$ & 0,8 & 1,0 \\
\hline 10 o más & 0,2 & 0,5 \\
\hline
\end{tabular}

\section{Diferencias en el uso de Internet en función del sexo y edad}

Considerando el uso de cualquiera de los servicios de Internet y para el grupo de jóvenes con un uso más frecuente de los mismos, los resultados mostraron que existen diferencias estadísticamente significativas a partir de los 18 años, edad en la que son los hombres los que invierten semanalmente un número de horas significativamente superior a las mujeres en el uso de la red. Concretamente, las diferencias porcentuales resultan ser significativas en el grupo de edad de 18 años (el $20 \%$ de los hombres frente al $8,2 \%$ de las mujeres, $p<0,05$ ), en el de 21 años (el $21,1 \%$ frente al $11,3 \%, p<0,05)$ y en el grupo de mayores de 21 años (el $20,2 \%$ de los hombres frente al 9,3\% de las mujeres, $p<0,01)$.

Al centrar el análisis en función del tipo de recurso preferido a la hora de conectarse, nuevamente, son los hombres los que hacen un uso más frecuente que las mujeres del correo electrónico, especialmente a la edad de 18 (el 15,2\% de los hombres frente al 6,2\% de las mujeres) y 20 años $(11,1 \%$ hombres frente al $3,9 \%$ ), y de las páginas web en los mismos rangos de edad $(28,3 \%$ hombres frente al $12,8 \%$ de mujeres y $35,7 \%$ de los hombres frente al $10,2 \%$ de mujeres, respectivamente)

Finalmente, en el acceso a líneas de chats, foros de discusión, Protocolos para Trasferencia de Ficheros y juegos en red no se encontraron diferencias estadísticamente significativas en su utilización en cuanto al sexo y la edad.

Interferencia percibida del uso de Internet en el desenvolvimiento cotidiano

El análisis de las respuestas de los universitarios de la muestra de estudio a los ítems dirigidos a evaluar la presencia de algún tipo de interferencia que el uso de Internet les había provocado en diversas áreas de la vida cotidiana, informó que sólo un porcentaje reducido de jóvenes (el 17\% de la muestra) percibe una influencia de esta naturaleza derivada de su implicación en la utilización de la red.

Concretamente, del conjunto de jóvenes que afirmaron percibir algún tipo de interferencia del uso de Internet en su vida cotidiana (230 sujetos), el 10,9\% informó haber descuidado sus obligaciones en más de una ocasión, el 3,6\% afirmó que su uso les había ocasionado problemas con su familia, el $2,4 \%$ de la muestra señaló que su trabajo y/o rendimiento se habían deteriorado desde que comenzaron a utilizar la red habitualmente y tan solo 9 universitarios percibían que el tiempo que empleaban en Internet les había hecho perder amistades.

\section{DISCUSIÓN}

Ante la creciente preocupación acerca de la capacidad de Internet para generar un proceso adictivo han ido surgiendo diferentes estudios en otros países dirigidos a analizar las posibles consecuencias negativas de su uso en determinados individuos. Parece necesario, quizás como paso previo a este tipo de análisis, comenzar la investigación a partir del análisis del uso específico que de este recurso se hace en nuestro país, puesto que sólo de esta forma podremos tomar conciencia de la magnitud del problema y de su posible abordaje.

Corroborando esta idea de la importancia de analizar el fenómeno del uso de Internet para avanzar en el desarrollo de modelos o propuestas de intervención de la posible patología derivada del mismo, se encuentran los resultados obtenidos en el presente estudio, que dibujan un panorama distinto del estado de la cuestión en comparación con el obtenido en otros países.

Si consideramos la magnitud del tiempo invertido en el uso de Internet, parámetro a través del cual se ha venido midiendo la existencia de una implicación patológica, los resultados obtenidos no parecen aproximarse a las proporciones descritas en otras investigaciones en las que la utilización de los servicios de la red sí parece ocupar un tiempo significativamente importante de la vida de los usuarios (Brenner, 1997). Así, de la muestra de estudio, más de la mitad de los universitarios afirman invertir menos de 4 horas semanales en Internet y tan solo el 3,7\% de los encuestados afirma dedicar entre 11-40 horas a la semana. Estos datos sí son más próximos a los obtenidos en 
otros estudios con población española en los que el número de usuarios que realizan un uso moderado de este recurso en cuanto al tiempo invertido en él, es significativamente superior al porcentaje muy reducido de internautas que dedican tiempos mucho más prolongados (según la AIMC en 1999, no superaban el 7\% de los encuestados).

Este hecho nos informa pues de que, en general, los estudiantes encuestados realizan un uso muy moderado de Internet y, aunque nos situemos en el grupo que dedica entre 11 y 20 horas semanales (lo que supone una implicación media al día de entre 1-3 horas), los datos obtenidos tampoco podrían indicarnos la existencia de un uso abusivo o patológico cercano a la adicción.

Tan sólo nos encontramos con un reducidísimo grupo de jóvenes con tiempos de conexión especialmente elevados (más de 40 horas, esto es, un mínimo de 5 horas diarias durante los 7 días de la semana) que sí presentan patrones de uso que podrían ser interpretados como problemáticos aunque sería necesario, sin lugar a dudas, estudiar en profundidad a este grupo de usuarios valorando el tipo de actividades que realizan en conexiones tan prolongadas. De cualquier forma, no debería olvidarse que en población española se trata de un número reducido de sujetos cuya situación, a la vista de los resultados, no podría generalizarse a toda la población de usuarios universitarios, como se ha venido haciendo desde otros países afirmando que éste sería uno los grupos poblacionales más susceptibles de generar problemas relacionados con el uso/abuso de Internet (Anderson, 1998).

Además, es necesario mencionar aquí que si analizamos la procedencia de los universitarios incluidos en la muestra de estudio (mayoritariamente de una institución privada), los resultados coincidirían con los obtenidos en diversos estudios previos que comparan el uso de Internet entre estudiantes de instituciones públicas y privadas. Estos trabajos señalan que éste es significativamente superior en el caso de los estudiantes de universidades públicas frente a los de privadas (afirman invertir menos de 3 horas semanales en la red, datos similares a los de nuestro estudio) y, además, presentarían un mayor número de consecuencias negativas derivadas del mismo (Davis et al., 1999).

El análisis de las diferencias en el uso de Internet en función de la edad y el sexo reveló la existencia de diferencias estadísticamente significativas en el uso de cualquiera de los servicios de Internet en los grupos de 18, 21 y más de 21 años; así como en el uso del correo electrónico, en los grupos de 18 y 20 años y en el acceso a páginas web en los grupos de 18, 20 y mayores de 21 años. En todos los casos anteriormente descritos fueron los hombres los que afirmaron invertir más tiempo a la semana en la utilización de cualquiera de esos recursos. Este hallazgo es con- sistente con investigaciones previas que muestran, además, la tendencia de los varones a presentar actitudes más positivas con respecto a Internet (Kraut et al., 1996; Morahan-Martin, 1998; Morahan-Martin y Schumacher, 2000; Sherman y cols., 2000).

Este hecho que evidencia el uso diferencial de los recursos de la red entre sexos ha sido interpretado en repetidas ocasiones en función de la evidencia observada desde hace años referente a la fascinación expresada preferentemente por los varones hacia las nuevas tecnologías, presentando un mejor dominio de los ordenadores tanto en lo referente al diseño de programas como al uso de otros recursos como los juegos de ordenador (De Santis y Youniss, 1991; McKenna, 1997; Schumacher y Morahan-Martin, 2001).

En definitiva, aunque a la luz de los resultados, el uso de Internet en población universitaria no parece alcanzar proporciones exageradas, es necesario considerar que su utilización se encuentra aún en fase de expansión en nuestro país y, si se analizan las tendencias observadas en otros países que sugieren que el uso de la red va a seguir incrementándose, los estudios específicos sobre los patrones de uso de la red se hacen cada vez más necesarios. Además, los escasos estudios existentes nos vuelven a recordar que es posible que la situación de los usuarios de nuestro país no se corresponda, por lo menos en el momento actual, con la problemática observada en otros países cuya familiarización con las nuevas tecnologías se ha realizado con mayor prontitud y desde hace más años.

De cualquier forma, será importante determinar en estudios posteriores qué tipo de variables caracterizan y diferencian a la minoría de usuarios que utilizan Internet durante tiempos muy prolongados y/o experimentan dificultades en su funcionamiento social, laboral o familiar por dicho uso, y que posiblemente puedan conformar el grupo de personas a las que sea necesario dirigir posteriores programas de intervención.

\section{BIBLIOGRAFÍA}

Anderson, K. (1998). Internet dependency among college students: should beconcerned?. Poster presentado en el encuentro de la American College Personnel Association, St. Louis, M.O.

Anderson, T.K., Law, S.A. y Mitchell, B.M. (1995). Universal access to E-mail: Feasability and Societal implication. Santa Mónica (CA): Rand Corporation.

Brenner, V. (1997). Psychology of computerr use: XLVII. Parameters of Internet use, abuse and addcition: the first 90 days of the internet usage survey. Psychological Reports, 80, 883-898. 
Davis, S.F., Smith, B., Rodrigue, K. y Pulvers, K. (1999). An examination of Internet usage on two college campuses. College Student Journal, 33, 2, 257-260.

De Santis, J. y Youniss, J. (1991). Family contributions to adolescent's attitudes toward new tecnology. Journal of Adolescent Research, 6, 410-422.

Echeburúa, E., Amor, P. y Cenea, R. (1998). Adicción a Internet: ¿una nueva adicción psicológica?. Monografías de Psiquiatría, 2, 38-44.

Estudio General de Medios: datos sobre Internet [EGM] (1999) [Online]. Disponible: http://www.aimc.es/ aimc/html/inter/net.html

FIND/SVP(1997). The 1997 American Internet User Survey. [Online]. Disponible: http://etrg.finsvp.com/findf/html

Griffits, M.D. (1997). Psychology of computer use: XLII. Some comments on addictive use of the internet by Young. Psychological Reports, 80, 81-82.

King, J.L. y y Kraemer, K.L. (1995). Information infraestructure, national policy, and global competitiveness. Information Infraestructure and Policy, 4, 5-28.

Kraut, R., Scherlis, W., Mukhopadhyay, T., Manning, J. y Kiesler, S. (1996). The HomeNet field trial of residential Internet services. Communications of the ACM, 39, 55-63.

Kraut, R., Patterson, M., Lundmark, V., Kiesler, S., Mukopadhyay, T. y Scherlis, W. (1998). Internet paradox: a social technology that reduces social involvement and psychological well-being?. American Psychologist, 53, 1017-1031.

McKenna, K. (1997). Dell identifies new generation without age and gender separation. Newsbites News Network. [Online]. Disponible: http://www.newsbytes.com
McKenna, K. y Bargh, J. (1998). Coming out in the age of the Internet: identity demarginalization throught virtual group participation. Journal of Personality and Social Psychology, 75 (3), 681-694.

Morahan-Martin, J. (1998). Males, females and the Internet. En J. Gackenback, Psychology and the Internet. San Diego: Academic Press.

Morahan-Martin, J. y Schumacher, P. (2000). Incidence and correlatesof pathological Internet use among college students. Computers in Human Behavior, 16, 13-29.

National Center for Educational Statistics [NCES] (1999). Condition of Education. [Online]. Disponible: http://www.nces.ed.gov/pubsinfo.asp?pubid=1999 02

NielsenNetRatings (1999). How many Online? [Online]. Disponible: http://www.nua.ie/surveys/how_many_ online/n_america.html

Parks, M. y Floyd, K. (1996). Making friends in cyberspace. Journal of Communication, 46 (1), 80-97.

Sherman, R.C., End, C., Kraan, E., Cole, A., Campbell, J., Birchmeier, Z. y Klausner, J. (2000). The Internet gender gap among college estudent: Forgotten but not gone? Cyberpsychology and Behavior, 3 (5), 885-894.

Schumaher, P. y Morahan-Martin, J. (2001). Gender, Internet and computer attitudes and experiencies. Computers in Human Behavior, 17, 95-110.

Tapscott, D. (1998). Growing up digital: the rise of the Net generation. New York: McGraw-Hill.

Young, K.S. (1998). Internet addiction: The emergence of a new clinical dosiorder. Cyberpsychology and Behavior, 1 (3), 237-244. 